1

2

3

\title{
How large are post-CCSD(T) contributions to the total atomization energies of medium-sized alkanes?
}

\author{
Amir Karton \\ School of Chemistry and Biochemistry, The University of Western Australia, Perth, WA 6009, \\ Australia
}

\begin{abstract}
A B S T RA C T
The CCSD(T) method is often considered as the gold standard in quantum chemistry for singlereference systems. Using $\mathrm{W} 4$ theory we calculate post-CCSD(T) contributions to the total atomization energies (TAEs) of $n$-alkanes and show that they reach up to $0.65 \mathrm{kcal} / \mathrm{mol}$ for $n$ hexane. Furthermore, we find that post-CCSD(T) contributions increase linearly with the size of the $n$-alkane, indicating that they will reach $\sim 1 \mathrm{kcal} / \mathrm{mol}$ for $n$-decane $\left(\mathrm{C}_{10} \mathrm{H}_{22}\right)$ and $\sim 2 \mathrm{kcal} / \mathrm{mol}$ for $n$-icosane $\left(\mathrm{C}_{20} \mathrm{H}_{42}\right)$. These results are significant since today CCSD(T)/CBS-type methods are being applied to hydrocarbons of increasing size and are assumed to give TAEs with chemical accuracy for these systems.
\end{abstract}

Keywords: CCSDTQ, CCSDT(Q), CCSDT, coupled cluster, W4 theory, W1 theory. 


\section{Introduction}

Application of coupled cluster theory involves two approximations. The first is the use of a finite basis set to express the orbitals in the Hartree-Fock wavefunction (a.k.a. the one-particle truncation error). The second is the truncation of the cluster operator to include only $n$-tuple excitations (a.k.a. the $n$-particle truncation error). ${ }^{1}$ Including all possible excitations in the wavefunction and carrying out the calculation with an infinitely large basis set will result in the exact nonrelativistic electronic energy, which is equivalent to a complete-basis-set fullconfiguration-interaction (FCI/CBS) calculation. A number of composite approaches for approximating the FCI/CBS energy have been developed in the past two decades. Prominent examples of such methods include the Weizmann-4 (W4), ${ }^{2-4}$ highly accurate extrapolated ab initio thermochemistry (HEAT), ${ }^{5,6}$ focal point approximation (FPA), ${ }^{7,8}$ and the so-called FellerPeterson-Dixon (FPD) ${ }^{9-12}$ methods. These theories have been shown to afford total atomization energies with confident sub-kJ mol ${ }^{-1}$ accuracy (i.e., $95 \%$ confidence intervals $<1 \mathrm{~kJ} \mathrm{~mol}^{-1}$, and maximal errors below $\sim 1 \mathrm{~kJ} \mathrm{~mol}^{-1}$ even for pathologically multireference systems such as ozone and halogen oxides). ${ }^{4,10,11,13-15}$

For systems dominated by a single reference determinant coupled cluster with singles, doubles, and quasiperturbative triple excitations (i.e., the $\operatorname{CCSD}(\mathrm{T})$ method) provides a very good approximation to the FCI energy at a significantly reduced computational cost. ${ }^{16,17}$ This is due to a systematic error cancellation between the higher-order connected triples, $T_{3}-(T)$, correlation contribution and the connected quadruple and quintuple excitations $\left(\mathrm{T}_{4}+\mathrm{T}_{5}\right)$. These contributions are of similar orders of magnitude, but the $T_{3}-(T)$ contribution tends to universally decrease the molecular binding energies, whereas the $T_{4}+T_{5}$ contribution universally increases them. This error compensation has been shown to work remarkably well for relatively small systems dominated by a single-reference configuration. ${ }^{2-6,18-22}$ The question that naturally arises, is how well this error compensation works for larger molecules? Or in other words, does the absolute magnitudes of the $T_{3}-(T)$ and $T_{4}+T_{5}$ contributions increase at the same rate or at different rates as the size of the system grows? This is a timely question, since with the increase in computational power of modern supercomputers and important theoretical developments, $\operatorname{CCSD}(\mathrm{T}) / \mathrm{CBS}$ composite methods are being applied to increasingly larger systems. Recent examples include the application of the W1-F12 composite method to systems as large as 

$\left(\mathrm{C}_{60}\right)^{24}$

In the present letter we will address this question for systems that are dominated by a single reference determinant, namely the homologous series of $n$-alkanes: methane, ethane, propane, butane, pentane, hexane, etc. We obtain the $\mathrm{T}_{3}-(\mathrm{T})$ and $\mathrm{T}_{4}+\mathrm{T}_{5}$ contributions for $n$ alkanes of up to hexane using W4 and W4lite theories. We find that close to the basis-set limit the error compensation between these two components becomes less and less effective with the size of the $n$-alkane, making the $\operatorname{CCSD}(\mathrm{T}) / \mathrm{CBS}$ method less and less accurate. In addition, we find that there is a nearly perfect linear correlation between the magnitude of the post-CCSD(T) and $\operatorname{CCSD}(\mathrm{T})$ correlation contributions, with a squared correlation coefficient of $\mathrm{R}^{2}>0.999$. We use this linear relationship to predict the post-CCSD(T) contributions for larger $n$-alkanes, and show that the post-CCSD(T) contribution is expected to exceed $1 \mathrm{kcal} \mathrm{mol}^{-1}$ for systems larger than decane $\left(\mathrm{C}_{10} \mathrm{H}_{22}\right)$.

\section{Computational details}

We obtain nonrelativistic total atomization energies (TAEs) for $n$-alkanes up to hexane using the W4lite and W4 composite procedures. ${ }^{2,4}$ The reference geometries for these calculations were optimized at the $\operatorname{CCSD}(\mathrm{T}) / \mathrm{cc}-\mathrm{pVQZ}$ level of theory as prescribed in the W4lite and W4 protocols. We note that throughout this work we use the so-called W4hlite and W4h versions of W4lite and W4 theories. The difference between the $\mathrm{W} n \mathrm{~h}$ protocols and the $\mathrm{Wn}$ protocols is that the diffuse functions are omitted from carbon in the SCF and valence CCSD(T) calculations. $^{21,25}$ For the hydrocarbon systems considered in the present work this approximation is of no thermochemical consequence, but computer resource requirements are substantially reduced. For the sake of simplicity we will simply refer to these theories as W4lite and W4.

For the sake of making the article self-contained, we will briefly outline the various steps in the W4lite and W4 procedures (for further details see Refs. 2 and 21). W4 theory represents a layered extrapolation to the CCSDTQ5 infinite basis set limit energy. The self-consistent field (SCF) energy is extrapolated from the cc-pV5Z and cc-pV6Z basis sets ${ }^{26}$ using the KartonMartin $^{27}$ modification of Jensen's extrapolation formula. ${ }^{28}$ The CCSD valence correlation energy is calculated from these same basis sets. Following the suggestion of Klopper, ${ }^{29}$ the CCSD correlation energy is partitioned into singlet- and triplet-coupled pair energies, which are 
extrapolated separately to the infinite basis set limit (see Ref. 2 for further details). The (T) valence correlation energy is extrapolated from the cc-pVQZ and cc-pV5Z basis sets. The higher-order connected triples, $\mathrm{T}_{3}-(\mathrm{T})$, correlation contribution is extrapolated from the cc-p $\mathrm{VnZ}$ basis sets $(n=\mathrm{D}$ and $\mathrm{T})$. The parenthetical connected quadruple, $(\mathrm{Q})$, and higher-order connected quadruple, $\mathrm{T}_{4}-(\mathrm{Q})$, correlation contributions are calculated with the cc-pVTZ and cc-pVDZ basis sets, respectively. The connected quintuple, $\mathrm{T}_{5}$, correlation contribution is calculated with the $s p$ part of the cc-pVDZ basis set (denoted by DZ).

In W4lite theory the post-CCSDT(Q) contributions are neglected altogether and the (Q) component is calculated in conjunction with the economical cc-pVDZ basis set. Nevertheless, for systems that are dominated by a single reference determinant, such as the hydrocarbons considered in the present work, W4lite gives very similar performance to W4. ${ }^{4}$

We also obtain the nonrelativistic TAEs for $n$-alkanes up to dodecane with the computationally efficient W1-F12 composite procedure. ${ }^{30} \mathrm{~W} 1-\mathrm{F} 12$ theory represents a layered extrapolation to the CCSD(T)/CBS energy. The computational protocol of the W1-F12 method has been specified in Ref. 30. In brief, the SCF energy is extrapolated from the cc-pVDZ-F12 and cc-pVTZ-F12 basis sets, ${ }^{31}$ which were developed for explicitly correlated calculations. The CCSD-F12 correlation energy is extrapolated from these same basis sets. The (T) valence correlation energy is obtained in the same way as in the original W1 theory, ${ }^{32}$ i.e., calculated with the standard $\operatorname{CCSD}(\mathrm{T})$ method and extrapolated to the basis set limit from the aug'-ccpVDZ and aug'-cc-pVTZ basis sets. ${ }^{33}$ The reference geometries used in the W1-F12 calculations were optimized at the B3LYP-D3BJ/aug'-cc-pVTZ level of theory ${ }^{34-37}$ as prescribed in the W1F12 protocol. ${ }^{30}$

All the $\operatorname{CCSD}(\mathrm{T})$ calculations were performed using the Molpro 2012.1 program suite. $^{38,39}$ All the post-CCSD(T) calculations were carried out with the MRCC program suite. ${ }^{40,41}$ All the B3LYP-D3BJ geometry optimizations were carried out using the Gaussian09 program suite. ${ }^{42}$

\section{Results and discussion}

Table 1 gives a component breakdown of the W4 atomization energies for the homologous series of $n$-alkanes: methane, ethane, propane, butane, pentane, and hexane. As might be expected there is a high statistical correlations between the SCF and CCSD and (T) correlation components and the number of carbon atoms in the $n$-alkanes. For example, the 
squared correlation coefficients $\left(\mathrm{R}^{2}\right)$ are: 1.0000 (SCF), 1.0000 (CCSD), and $0.9998((\mathrm{~T}))$. We note that high statistical correlations have also been observed for the SCF, CCSD and (T) components of the TAEs in other homologous series. For example, the series of platonic hydrocarbon cages (tetrahedrane, triprismane, cubane, pentaprismane, octahedrane, and dodecahedrane $)^{23}$ and the series of linear polyacenes (benzene, naphthalene, anthracene, and naphthacene). ${ }^{30}$

Table 1. Component breakdown of the $\mathrm{W} 4$ and $\mathrm{W} 4$ lite valence, nonrelativistic atomization energies for $n$-alkanes of up to hexane (in $\mathrm{kcal} \mathrm{mol}^{-1}$ ).

\begin{tabular}{lcccccccc}
\hline & $\mathrm{SCF}^{a}$ & $\mathrm{CCSD}^{a}$ & $(\mathrm{~T})^{b}$ & $\mathrm{~T}_{3}-(\mathrm{T})^{c}$ & $\mathrm{~T}_{4}{ }^{d}$ & $\mathrm{~T}_{4}{ }^{e}$ & $\mathrm{~T}_{5}{ }^{2}$ & $\mathrm{~T}_{4}+\mathrm{T}_{5}{ }^{g}$ \\
& $\mathrm{~W} 4$ & $\mathrm{~W} 4$ & $\mathrm{~W} 4$ & $\mathrm{~W} 4$ & $\mathrm{~W} 4$ lite & $\mathrm{W} 4$ & $\mathrm{~W} 4$ & $\mathrm{~W} 4$ \\
\hline $\mathrm{CH}_{4}{ }^{h}$ & 331.546 & 84.737 & 2.890 & -0.080 & 0.079 & 0.080 & 0.000 & 0.080 \\
$\mathrm{C}_{2} \mathrm{H}_{6}{ }^{h}$ & 558.015 & 146.340 & 6.370 & -0.350 & 0.219 & 0.230 & 0.010 & 0.240 \\
$\mathrm{C}_{3} \mathrm{H}_{8}{ }^{h}$ & 785.335 & 209.050 & 10.120 & -0.630 & 0.355 & 0.380 & $0.019^{i}$ & 0.399 \\
$\mathrm{C}_{4} \mathrm{H}_{10}$ & 1012.593 & 271.812 & 13.883 & -0.930 & 0.530 & 0.572 & $0.026^{i}$ & 0.597 \\
$\mathrm{C}_{5} \mathrm{H}_{12}$ & 1239.834 & 334.541 & 17.654 & -1.227 & 0.688 & N/A & N/A & $0.787^{j}$ \\
$\mathrm{C}_{6} \mathrm{H}_{14}$ & 1467.005 & 397.384 & 21.441 & $-1.498^{k}$ & 0.847 & N/A & N/A & N/A \\
$\mathrm{R}^{2 l}$ & 1.0000 & 1.0000 & 0.9998 & 0.9997 & 0.9985 & 0.9961 & 0.9923 & 0.9974
\end{tabular}

${ }^{a}$ The SCF and valence CCSD correlation components are extrapolated from the cc-pV5Z and cc-pV6Z basis sets. ${ }^{b}$ The valence (T) correlation component is extrapolated from the cc-pVQZ and cc-pV5Z basis sets. ${ }^{c}$ The valence CCSDT-CCSD(T) correlation component extrapolated from the cc-pVDZ and cc-pVTZ basis sets. ${ }^{d}$ The $\mathrm{T}_{4}$ component is taken as at the $(\mathrm{Q}) / \mathrm{cc}-\mathrm{pVDZ}$ correlation component. ${ }^{e}$ The CCSDT(Q)-CCSDT component is calculated with the cc-pVTZ basis set, whilst the CCSDTQ-CCSDT(Q) component is calculated with the cc-pVDZ basis set (see Ref. 2 for further details). ${ }^{f}$ The CCSDTQ5-CCSDTQ component is calculated with the DZ basis set. ${ }^{g}$ Sum of the $\mathrm{T}_{4}+\mathrm{T}_{5}$ contributions from W4 theory. ${ }^{h}$ Taken from ref. ${ }^{21} .{ }^{i} \mathrm{CCSDTQ}(5)-\mathrm{CCSDTQ}$ component calculated with the DZ basis set. ${ }^{j}$ The $\mathrm{T}_{4}+\mathrm{T}_{5}$ contribution is estimated assuming that the post-CCSD(T) contribution to the isodesmic reaction $\mathrm{C}_{5} \mathrm{H}_{12}+3 \mathrm{CH}_{4} \rightarrow 4 \mathrm{C}_{2} \mathrm{H}_{6}$ are zero and taking the $\mathrm{T}_{3}-(\mathrm{T})$ contribution from W4 theory. ${ }^{k}$ The $\mathrm{T}_{3}-(\mathrm{T})$ contribution is estimated assuming that the post-CCSD(T) contribution to the isodesmic reaction $\mathrm{C}_{6} \mathrm{H}_{14}+4 \mathrm{CH}_{4} \rightarrow$

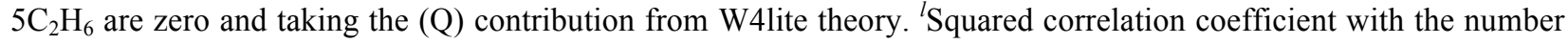
of carbon atoms in the $n$-alkanes.

What about the correlation between the SCF, CCSD, and (T) components of the TAEs and the number of carbon atoms in larger $n$-alkanes? We were able to obtain these components from W1-F12 for n-alkanes of up to dodecane. Table 2 lists these components of the TAEs along with the $\mathrm{R}^{2}$ values. There is perfect linear correlation between these components and the number 
of carbons in the $n$-alkanes. Namely, for the SCF, CCSD, and (T) components we obtain $\mathrm{R}^{2}=$ 1.0000 .

Table 2. Component breakdown of the W1-F12 valence, nonrelativistic atomization energies for $n$-alkanes of up to dodecane (in $\mathrm{kcal} \mathrm{mol}^{-1}$ ).

\begin{tabular}{lcccc}
\hline & $\mathrm{SCF}^{a}$ & $\mathrm{CCSD}^{a}$ & $(\mathrm{~T})^{b}$ & $\mathrm{CCSD}(\mathrm{T})^{c}$ \\
\hline $\mathrm{CH}_{4}$ & 331.51 & 84.58 & 2.92 & 87.51 \\
$\mathrm{C}_{2} \mathrm{H}_{6}$ & 557.95 & 146.20 & 6.42 & 152.63 \\
$\mathrm{C}_{3} \mathrm{H}_{8}$ & 785.30 & 208.83 & 10.16 & 218.98 \\
$\mathrm{C}_{4} \mathrm{H}_{10}$ & 1012.56 & 271.57 & 13.93 & 285.49 \\
$\mathrm{C}_{5} \mathrm{H}_{12}$ & 1239.77 & 334.33 & 17.70 & 352.03 \\
$\mathrm{C}_{6} \mathrm{H}_{14}$ & 1466.98 & 397.11 & 21.49 & 418.59 \\
$\mathrm{C}_{7} \mathrm{H}_{16}$ & 1694.20 & 459.89 & 25.27 & 485.16 \\
$\mathrm{C}_{8} \mathrm{H}_{18}$ & 1921.41 & 522.67 & 29.06 & 551.72 \\
$\mathrm{C}_{9} \mathrm{H}_{20}$ & 2148.62 & 585.45 & 32.84 & 618.29 \\
$\mathrm{C}_{10} \mathrm{H}_{22}$ & 2375.85 & 648.22 & 36.63 & 684.85 \\
$\mathrm{C}_{11} \mathrm{H}_{24}$ & 2603.07 & 711.00 & 40.41 & 751.41 \\
$\mathrm{C}_{12} \mathrm{H}_{26}$ & 2830.29 & 773.78 & 44.20 & 817.98 \\
$\mathrm{R}^{2 d}$ & 1.0000 & 1.0000 & 1.0000 & 1.0000
\end{tabular}

${ }^{a}$ The SCF and valence CCSD-F12 correlation components are extrapolated from the cc-pVDZ-F12 and cc-pVDZ-F12 basis sets. ${ }^{b}$ The valence (T) correlation component is extrapolated from the cc-pVDZ and cc-pVTZ basis sets. ${ }^{c}$ Sum of the CCSD-F12 and (T) correlation components. ${ }^{d}$ Squared correlation coefficient with the number of carbon atoms in the $n$-alkanes.

In the present work we would like to explore the linear correlation between the post$\operatorname{CCSD}(\mathrm{T})$ correlation components of the TAE and the number of carbon atoms in the $n$-alkanes. However, first a few comments are due on the magnitude of the post-CCSD(T) contributions. These results are shown in Table 1 . The higher-order connected triple, $\mathrm{T}_{3}-(\mathrm{T})$, contributions universally reduce the atomization energies and range between $-0.080\left(\mathrm{CH}_{4}\right)$ and $-1.498\left(\mathrm{C}_{6} \mathrm{H}_{14}\right)$ $\mathrm{kcal} \mathrm{mol}^{-1}$. The parenthetical connected quadruple contributions from W4lite theory universally increase the atomization energies and range between $0.079\left(\mathrm{CH}_{4}\right)$ and $0.847\left(\mathrm{C}_{6} \mathrm{H}_{14}\right) \mathrm{kcal} \mathrm{mol}^{-1}$. We were able to obtain the full quadruple contributions from W4 theory (i.e., including higherorder connected quadruple, $\mathrm{T}_{4}-(\mathrm{Q})$, contributions) for $n$-alkanes up to butane. There is 

$\mathrm{I}_{4}$ contributions from W4 theory (Table 1). Specifically, the $\mathrm{T}_{4}$ values from W4lite underestimate those from W4 by $0.001\left(\mathrm{CH}_{4}\right), 0.011\left(\mathrm{C}_{2} \mathrm{H}_{6}\right), 0.025\left(\mathrm{C}_{3} \mathrm{H}_{8}\right)$, and $0.042\left(\mathrm{C}_{4} \mathrm{H}_{10}\right)$

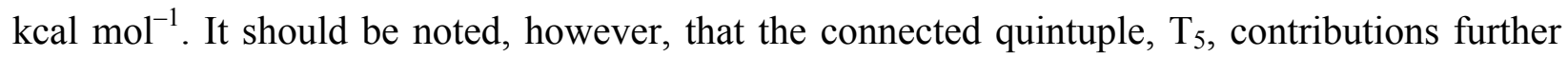
increase the gap between W4lite and W4 by $0.000\left(\mathrm{CH}_{4}\right), 0.010\left(\mathrm{C}_{2} \mathrm{H}_{6}\right), 0.019\left(\mathrm{C}_{3} \mathrm{H}_{8}\right)$, and 0.026 $\left(\mathrm{C}_{4} \mathrm{H}_{10}\right) \mathrm{kcal} \mathrm{mol}^{-1}$. Overall, W4lite theory underestimates the W4 post-CCSDT components by: $0.001\left(\mathrm{CH}_{4}\right), 0.021\left(\mathrm{C}_{2} \mathrm{H}_{6}\right), 0.044\left(\mathrm{C}_{3} \mathrm{H}_{8}\right)$, and $0.067\left(\mathrm{C}_{4} \mathrm{H}_{10}\right) \mathrm{kcal} \mathrm{mol}{ }^{-1}$. Thus, it seems that these difference increase by $\sim 0.02 \mathrm{kcal} \mathrm{mol}^{-1}$ per each additional carbon atom in the $n$-alkane.

Let us now turn to the magnitude of the overall post-CCSD(T) contributions to the TAEs (i.e., the sum of the $T_{3}-(T)+T_{4}+T_{5}$ contributions). Table 3 lists these contributions from W4lite and $\mathrm{W} 4$ theories. Overall the post-CCSD(T) contributions tend systematically decreases the TAEs. Let us begin with the $\mathrm{W} 4$ results. For methane there is a perfect cancellation between the $\mathrm{T}_{3}-(\mathrm{T})$ and $\mathrm{T}_{4}+\mathrm{T}_{5}$ components, such that the overall post-CCSD(T) contribution to the TAE is essentially zero. For ethane there is still a very effective cancellation between the $T_{3}-(T)$ and $T_{4}$ $+\mathrm{T}_{5}$ terms, namely the overall post-CCSD(T) contribution amounts to $-0.110 \mathrm{kcal}^{\mathrm{mol}}{ }^{-1}$. However, for propane the cancellation between the $T_{3}-(T)$ and $T_{4}+T_{5}$ components is less effective with an overall post-CCSD(T) contribution of $-0.231 \mathrm{kcal} \mathrm{mol}^{-1}$, and this cancelation becomes less and less effective with the size of the system. The overall post-CCSD(T) contributions to the TAE are -0.332 (butane) and -0.440 (pentane) $\mathrm{kcal} \mathrm{mol}^{-1}$. In fact, there is a nearly perfect linear correlation between the magnitude of the post-CCSD(T) contributions and the number of carbon atoms in the $n$-alkane $\left(\mathrm{R}^{2}=0.9993\right.$, Table 3$)$. This is a result of the fact that both the $\mathrm{T}_{3}-(\mathrm{T})$ and $\mathrm{T}_{4}+\mathrm{T}_{5}$ components correlate linearly with the number of carbon atoms (Table 1), however, the $T_{3}-(T)$ contribution decreases at a faster rate than the $T_{4}+T_{5}$ contribution increases. This is illustrated in Figure 1a. Specifically, the overall post-CCSD(T) contribution to the TAE increases by about $0.1 \mathrm{kcal} \mathrm{mol}^{-1}$ per carbon atom. 
Table 3. Overall post-CCSD(T) contributions to the atomization energies of $n$-alkanes up to hexane from $\mathrm{W} 4$ and W4lite theories (in $\mathrm{kcal} \mathrm{mol}^{-1}$ ).

\begin{tabular}{lcc}
\hline & W4lite $^{a}$ & W4 $^{b}$ \\
\hline $\mathrm{CH}_{4}$ & -0.001 & 0.000 \\
$\mathrm{C}_{2} \mathrm{H}_{6}$ & -0.131 & -0.110 \\
$\mathrm{C}_{3} \mathrm{H}_{8}$ & -0.275 & -0.231 \\
$\mathrm{C}_{4} \mathrm{H}_{10}$ & -0.400 & -0.332 \\
$\mathrm{C}_{5} \mathrm{H}_{12}$ & -0.539 & -0.440 \\
$\mathrm{C}_{6} \mathrm{H}_{14}$ & -0.651 & N/A \\
$\mathrm{R}^{2 c}$ & 0.9991 & 0.9993
\end{tabular}

${ }^{a}$ Sum of the $\mathrm{T}_{3}-(\mathrm{T})+\mathrm{T}_{4}$ contributions from W4lite theory. ${ }^{b} \mathrm{Sum}$ of the $\mathrm{T}_{3}-(\mathrm{T})+\mathrm{T}_{4}+\mathrm{T}_{5}$ contributions from W4 theory. ${ }^{c}$ Squared correlation coefficient with the number of carbon atoms in the $n$-alkane.
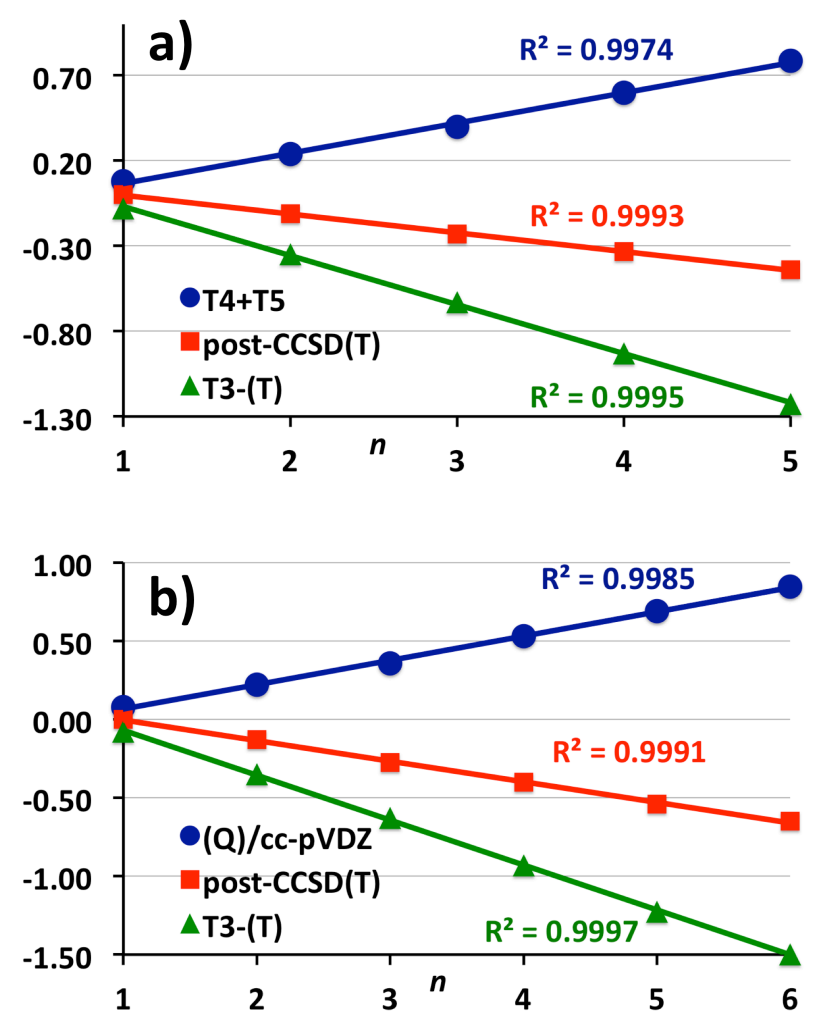

Figure 1. Linear correlation between the $\mathrm{T}_{3}-(\mathrm{T})$, post-CCSDT, and post-CCSD $(\mathrm{T})$ components to the TAEs (in $\mathrm{kcal} \mathrm{mol}^{-1}$ ) and the number of carbons in the $\mathrm{C}_{n} \mathrm{H}_{2 n+2}$ straight-chain alkanes from (a) W4 theory and (b) W4lite theory. 
We were able to obtain the post-CCSD(T) contributions to the TAEs from W4lite theory for $n$-alkanes of up to hexane (Table 3 and Figure 1b). The W4lite results show that the $\operatorname{CCSD}(\mathrm{T}) / \mathrm{CBS}$ level of theory will have an error of $0.65 \mathrm{kcal} \mathrm{mol}^{-1}$ for $n$-hexane due to the neglect of post-CCSD $(\mathrm{T})$ correlation effects.

In the preceding paragraphs we have shown that the post-CCSD(T) contribution correlates linearly with the number of carbon atoms in $n$-alkanes up to hexane (Tables 1 and 3), and that the $\operatorname{CCSD}(\mathrm{T})$ correlation component correlates linearly with the number of carbon atoms in $n$-alkanes up to dodecane (Tables 1 and 2). It is instructive to see what is the correlation between the $\operatorname{CCSD}(\mathrm{T})$ and post-CCSD $(\mathrm{T})$ correlation components. For $n$-alkanes up to pentane we obtain a squared correlation coefficient of $\mathrm{R}^{2}=0.9992$ between the $\operatorname{CCSD}(\mathrm{T})$ and post$\operatorname{CCSD}(\mathrm{T})$ correlation components from W4 theory. Whilst for $n$-alkanes up to hexane we obtain $\mathrm{R}^{2}=0.9990$ between the $\operatorname{CCSD}(\mathrm{T})$ and post-CCSD $(\mathrm{T})$ correlation components from W4lite theory. We can use these linear relationships to predict the post-CCSD(T) correlation contributions for larger $n$-alkanes using the $\operatorname{CCSD}(\mathrm{T})$ correlation component calculated from W1-F12 theory (Table 2). Table 4 gives these results for $n$-alkanes of up to dodecane $\left(\mathrm{C}_{12} \mathrm{H}_{26}\right)$. We first note that, for the $n$-alkanes for which we have W4lite and W4 data, there is excellent agreement between the estimated and actual post- $\operatorname{CCSD}(\mathrm{T})$ contributions. In particular, the deviations are generally smaller than $0.01 \mathrm{kcal} \mathrm{mol}^{-1}$ (Tables 3 and 4). We also note that the post-CCSD(T) contributions estimated from the W4lite data systematically overestimate those estimated from the W4 data (Table 4). This is consistent with the results from W4lite and W4 theories for the smaller $n$-alkanes (Table 3). 
Table 4. Post-CCSD(T) contributions to the atomization energies of $n$-alkanes up to dodecane estimated from the linear relationship between the $\operatorname{CCSD}(\mathrm{T})$ and post-CCSD $(\mathrm{T})$ correlation components in W4lite and W4 theories (in $\mathrm{kcal} \mathrm{mol}^{-1}$ ).

\begin{tabular}{lcc}
\hline & W4lite $^{a}$ & W4 $^{b}$ \\
\hline $\mathrm{CH}_{4}$ & -0.01 & 0.00 \\
$\mathrm{C}_{2} \mathrm{H}_{6}$ & -0.13 & -0.11 \\
$\mathrm{C}_{3} \mathrm{H}_{8}$ & -0.27 & -0.22 \\
$\mathrm{C}_{4} \mathrm{H}_{10}$ & -0.40 & -0.33 \\
$\mathrm{C}_{5} \mathrm{H}_{12}$ & -0.53 & -0.44 \\
$\mathrm{C}_{6} \mathrm{H}_{14}$ & -0.66 & -0.55 \\
$\mathrm{C}_{7} \mathrm{H}_{16}$ & -0.79 & -0.66 \\
$\mathrm{C}_{8} \mathrm{H}_{18}$ & -0.92 & -0.78 \\
$\mathrm{C}_{9} \mathrm{H}_{20}$ & -1.06 & -0.89 \\
$\mathrm{C}_{10} \mathrm{H}_{22}$ & -1.19 & -1.00 \\
$\mathrm{C}_{11} \mathrm{H}_{24}$ & -1.32 & -1.11 \\
$\mathrm{C}_{12} \mathrm{H}_{26}$ & -1.45 & -1.22
\end{tabular}

${ }^{a}$ The linear relationship between the $\operatorname{CCSD(T)}$ and post-CCSD(T) correlation components is obtained from W4lite theory for $n$-alkanes up to hexane, specifically it is post-CCSD(T) $=-0.00198 \times \operatorname{CSSD}(\mathrm{T})^{\text {corr }}+0.16779\left(\mathrm{R}^{2}=0.9990\right)$. ${ }^{c}$ The linear relationship between the $\operatorname{CCSD}(\mathrm{T})$ and post-CCSD(T) correlation components is obtained from W4 theory for $n$-alkanes up to pentane, specifically it is post-CCSD $(\mathrm{T})=-0.00166 \times \operatorname{CSSD}(\mathrm{T})^{\mathrm{corr}}+0.14271\left(\mathrm{R}^{2}=\right.$ 0.9992). ${ }^{c} \mathrm{The} \operatorname{CCSD}(\mathrm{T})$ correlation components used for obtaining the post-CCSD(T) correlation components for $\mathrm{C}_{n} \mathrm{H}_{2 n+2}(n=1-12)$ are taken from W1-F12 theory (Table 2).

Perhaps the most striking result of Table 4 is that the overall post-CCSD(T) contribution to the TAE is expected to exceed the $1 \mathrm{kcal} \mathrm{mol}^{-1}$ mark for systems larger than $\mathrm{C}_{10} \mathrm{H}_{22}$. According to our W4 and W1-F12 data it will reach $1.2 \mathrm{kcal} \mathrm{mol}^{-1}$ for $\mathrm{C}_{12} \mathrm{H}_{26}$. In addition, we can estimate the magnitude of the post-CCSD(T) contribution for $n$-alkanes larger than $\mathrm{C}_{12} \mathrm{H}_{26}$ by exploiting the high statistical correlation $\left(\mathrm{R}^{2}=0.9993\right)$ between the post-CCSD $(\mathrm{T})$ contribution and the number of carbons in the $n$-alkane (Table 3). From linear regression fits to the W4 data we estimate that the post-CCSD(T) contribution for icosane $\left(\mathrm{C}_{20} \mathrm{H}_{42}\right)$ will exceed 2 kcal mol ${ }^{-1}$. 


\section{Conclusions}

We examine the post-CCSD(T) correlation contributions to the TAEs of straight-chain alkanes using the W4lite and W4 composite procedures. Using the more rigorous W4 theory we obtain the following post-CCSD(T) contributions to the TAEs: $0.000\left(\mathrm{CH}_{4}\right),-0.110\left(\mathrm{C}_{2} \mathrm{H}_{6}\right)$, $0.231\left(\mathrm{C}_{3} \mathrm{H}_{8}\right),-0.332\left(\mathrm{C}_{4} \mathrm{H}_{10}\right)$, and $-0.440\left(\mathrm{C}_{5} \mathrm{H}_{12}\right) \mathrm{kcal} \mathrm{mol}^{-1}$. Whilst W4lite theory gives somewhat larger post-CCSD(T) contributions, namely: $-0.001\left(\mathrm{CH}_{4}\right),-0.131\left(\mathrm{C}_{2} \mathrm{H}_{6}\right),-0.275$ $\left(\mathrm{C}_{3} \mathrm{H}_{8}\right),-0.400\left(\mathrm{C}_{4} \mathrm{H}_{10}\right),-0.539\left(\mathrm{C}_{5} \mathrm{H}_{12}\right)$, and $-0.651\left(\mathrm{C}_{6} \mathrm{H}_{14}\right) \mathrm{kcal} \mathrm{mol}^{-1}$. This gradual increase in the magnitude of the post-CCSD(T) correlation contribution is a result of a small but systematic imbalance in the rate at which the $\mathrm{T}_{3}-(\mathrm{T})$ and $\mathrm{T}_{4}+\mathrm{T}_{5}$ contributions increase with the size of the system. In particular, the $T_{3}-(T)$ contribution, which tends to decrease the TAE, increase faster with the size of the system than the $T_{4}+T_{5}$ contributions, which tend to universally increase the TAE. Overall, the post-CCSD(T) contributions increase linearly with the size of the system, by about $0.1 \mathrm{kcal} \mathrm{mol}^{-1}$ per each additional carbon atom. Small as this number may seem in relative terms, it means that the intrinsic error of the $\operatorname{CCSD}(\mathrm{T}) / \mathrm{CBS}$ atomization energy for decane $\left(\mathrm{C}_{10} \mathrm{H}_{22}\right)$ is expected to approach $\sim 1 \mathrm{kcal} \mathrm{mol}^{-1}$, and $\sim 2 \mathrm{kcal} \mathrm{mol}^{-1}$ for icosane $\left(\mathrm{C}_{20} \mathrm{H}_{42}\right)$. These results are surprising since $n$-alkanes are dominated by a single reference determinant and it is generally accepted that the $\mathrm{CCD}(\mathrm{T}) / \mathrm{CBS}$ method should give atomization energies within chemical accuracy for these systems (i.e., with deviations below $1 \mathrm{kcal} \mathrm{mol}^{-1}$ from $\mathrm{FCI} / \mathrm{CBS}$ reference values).

\section{Supplementary data}

$\operatorname{CCSD}(\mathrm{T}) / \mathrm{cc}-\mathrm{pVQZ}$ optimized geometries for $n$-alkanes up to hexane (Table S1) and B3LYPD3BJ/aug'-cc-pVTZ optimized geometries for $n$-alkanes up to dodecane (Table S2).

\section{Corresponding author}

*E-Mail: amir.karton@chem.usyd.edu.au

\section{Acknowledgments}

We gratefully acknowledge the generous allocation of computing time from the National Computational Infrastructure (NCI) National Facility, and system administration support 
provided by the Faculty of Science at UWA to the Linux cluster of the Karton group. AK is the recipient of an Australian Research Council (ARC) Discovery Early Career Researcher Award (DECRA, project number: DE140100311).

\section{References}

(1) Shavitt, I.; Bartlett, R. J. Many-body methods in chemistry and physics: MBPT and coupled-cluster theory; Cambridge university press, 2009.

(2) Karton, A.; Rabinovich, E.; Martin, J. M. L.; Ruscic, B. Journal of Chemical Physics 2006, 125, 144108.

(3) Karton, A.; Taylor, P. R.; Martin, J. M. L. Journal of Chemical Physics 2007, $127,064104$.

(4) Karton, A.; Daon, S.; Martin, J. M. L. Chemical Physics Letters 2011, 510, 165.

(5) Bomble, Y. J.; Vazquez, J.; Kallay, M.; Michauk, C.; Szalay, P. G.; Csaszar, A. G.; Gauss, J.; Stanton, J. F. J Chem Phys 2006, 125, 64108.

(6) Harding, M. E.; Vazquez, J.; Ruscic, B.; Wilson, A. K.; Gauss, J.; Stanton, J. F. J Chem Phys 2008, 128, 114111.

(7) Allen, W. D.; East, A. L.; Csaszar, A. G. In Structures and Conformations of Non-Rigid Molecules; Springer Netherlands: 1993, p 343.

(8) Csaszar, A. G.; Allen, W. D.; Schaefer III, H. F. Journal of Chemical Physics 1998, $108,9751$.

(9) Feller, D.; Peterson, K. A.; Dixon, D. A. J Chem Phys 2008, 129, 204105.

(10) Peterson, K. A.; Feller, D.; Dixon, D. A. Theoretical Chemistry Accounts 2012, $131,1079$.

(11) Feller, D.; Peterson, K. A.; Dixon, D. A. Molecular Physics 2012, 110, 2381.

(12) Feller, D.; Peterson, K. A.; Ruscic, B. Theoretical Chemistry Accounts 2013, 133, 1407.

(13) Feller, D.; Peterson, K. A.; Dixon, D. A. The Journal of Physical Chemistry A 2009, 114,613 .

(14) Karton, A.; Parthiban, S.; Martin, J. The Journal of Physical Chemistry A 2009, 113,4802 .

(15) Helgaker, T.; Klopper, W.; Tew, D. P. Molecular Physics 2008, 106, 2107. 
(16) Raghavachari, K.; Trucks, G. W.; Pople, J. A.; Head-Gordon, M. Chem. Phys. Lett. 1989, 157479.

(17) Watts, J. D.; Gauss, J.; Bartlett, R. J. J. Chem. Phys. 1993, 98, 8718.

(18) Helgaker, T.; Klopper, W.; Halkier, A.; Bak, K.; Jørgensen, P.; Olsen, J. In Quantum-Mechanical Prediction of Thermochemical Data; Cioslowski, J., Ed.; Springer Netherlands: 2001; Vol. 22, p 1.

(19) Tajti, A.; Szalay, P. G.; Császár, A. G.; Kállay, M.; Gauss, J.; Valeev, E. F.; Flowers, B. A.; Vázquez, J.; Stanton, J. F. The Journal of chemical physics 2004, 121, 11599.

(20) Boese, A. D.; Oren, M.; Atasoylu, O.; Martin, J. M. L.; Kállay, M.; Gauss, J. Journal of chemical physics 2004, 120, 4129.

(21) Karton, A.; Gruzman, D.; Martin, J. M. L. The Journal of Physical Chemistry A 2009, 113, 8434 .

(22) Oyeyemi, V. B.; Pavone, M.; Carter, E. A. ChemPhysChem 2011, 12, 3354.

(23) Karton, A.; Schreiner, P. R.; Martin, J. M. L. Journal of Computational Chemistry 2016, 37, 49 .

(24) Karton, A.; Wan, W. Chemical Physics Letters 2016, 64334.

(25) Martin, J. M. L.; Parthiban, S. In Quantum-Mechanical Prediction of Thermochemical Data; Cioslowski, J., Ed.; Springer Netherlands: 2001; Vol. 22, p 31.

(26) Dunning Jr, T. H. J Chem Phys 1989, 90, 1007.

(27) Karton, A.; Martin, J. M. L. Theoretical Chemistry Accounts 2006, 115, 330.

(28) Jensen, F. Theoretical Chemistry Accounts 2005, 113, 267.

(29) Klopper, W. Molecular Physics 2001, 99, 481.

(30) Karton, A.; Martin, J. M. L. Journal of Chemical Physics 2012, 136, 124114.

(31) Peterson, K. A.; Adler, T. B.; Werner, H.-J. J Chem Phys 2008, 128, 084102.

(32) Martin, J. M. L.; de Oliveira, G. J Chem Phys 1999, 111, 1843.

(33) The notation aug'-cc-pVnZ indicates the combination of the standard correlationconsistent cc-pVnZ basis sets on hydrogen, and the aug-cc-pVnZ basis sets on carbon.

(34) Becke, A. D. Journal of Chemical Physics 1993, 98, 1372.

(35) Lee, C. T.; Yang, W. T.; Parr, R. G. Physical Review B 1988, 37, 785.

(36) Grimme, S.; Antony, J.; Ehrlich, S.; Krieg, H. Journal of Chemical Physics 2010, $132,19$. 

$32,1456$. H.-J. Werner; P. J. Knowles; G. Knizia; F. R. Manby; M. Schütz; P. Celani; T. Korona; R. Lindh; A. Mitrushenkov; G. Rauhut; K. R. Shamasundar; T. B. Adler; R. D. Amos; A. Bernhardsson; A. Berning; D. L. Cooper; M. J. O. Deegan; A. J. Dobbyn; F. Eckert; E. Goll; C. Hampel; A. Hesselmann; G. Hetzer; T. Hrenar; G. Jansen; C. Köppl; Y. Liu; A. W. Lloyd; R. A. Mata; A. J. May; S. J. McNicholas; W. Meyer; M. E. Mura; A. Nicklass; D. P. O'Neill; P. Palmieri; D. Peng; K. Pflüger; R. Pitzer; M. Reiher; T. Shiozaki; H. Stoll; A. J. Stone; R. Tarroni; T. Thorsteinsson; Wang, M. MOLPRO, version 2012.1, a package of ab initio programs.

(39) Werner, H.-J.; Knowles, P. J.; Knizia, G.; Manby, F. R.; Schütz, M. Wiley Interdisciplinary Reviews: Computational Molecular Science 2012, 2, 242.

(40) Kállay, M.; Rolik, Z.; Csontos, J.; Ladjánszki, I.; Szegedy, L.; Ladóczki, B.; Samu, G. MRCC, a quantum chemical program suite. See also: http://www.mrcc.hu.

(41) Rolik, Z.; Szegedy, L.; Ladjánszki, I.; Ladóczki, B.; Kállay, M. The Journal of chemical physics 2013, 139, 094105.

(42) Frisch, M. J.; Trucks, G. W.; Schlegel, H. B.; Scuseria, G. E.; Robb, M. A.; Cheeseman, J. R.; Scalmani, G.; Barone, V.; Mennucci, B.; Petersson, G. A.; Nakatsuji, H.; Caricato, M.; Li, X.; Hratchian, H. P.; Izmaylov, A. F.; Bloino, J.; Zheng, G.; Sonnenberg, J. L.; Hada, M.; Ehara, M.; Toyota, K.; Fukuda, R.; Hasegawa, J.; Ishida, M.; Nakajima, T.; Honda, Y.; Kitao, O.; Nakai, H.; Vreven, T.; Montgomery Jr., J. A.; Peralta, J. E.; Ogliaro, F.; Bearpark, M. J.; Heyd, J.; Brothers, E. N.; Kudin, K. N.; Staroverov, V. N.; Kobayashi, R.; Normand, J.; Raghavachari, K.; Rendell, A. P.; Burant, J. C.; Iyengar, S. S.; Tomasi, J.; Cossi, M.; Rega, N.; Millam, N. J.; Klene, M.; Knox, J. E.; Cross, J. B.; Bakken, V.; Adamo, C.; Jaramillo, J.; Gomperts, R.; Stratmann, R. E.; Yazyev, O.; Austin, A. J.; Cammi, R.; Pomelli, C.; Ochterski, J. W.; Martin, R. L.; Morokuma, K.; Zakrzewski, V. G.; Voth, G. A.; Salvador, P.; Dannenberg, J. J.; Dapprich, S.; Daniels, A. D.; Farkas, Ö.; Foresman, J. B.; Ortiz, J. V.; Cioslowski, J.; Fox, D. J.; Gaussian, Inc.: Wallingford, CT, USA, 2009. 